\title{
Survey of Taurine Uptake and Metabolism in Staphylococcus aureus
}

\author{
By DAVID W. SMILEY† AND BRIAN J. WILKINSON* \\ Microbiology Group, Department of Biological Sciences, Illinois State University, Normal, \\ Illinois 61761, U.S.A.
}

(Received 21 December 1982; revised 22 March 1983)

\begin{abstract}
Taurine has been reported to be a component of the capsular polysaccharide of the encapsulated M strain of Staphylococcus aureus. This led to a study of the uptake and metabolism of [1,2${ }^{14} \mathrm{C}$ ]taurine in a variety of encapsulated and unencapsulated $S$. aureus strains. Taurine was taken up by all strains studied. A discrepancy between uptake measured as depletion of radioactivity from growth medium and as cell-associated radioactivity suggested that taurine may be catabolized to $\mathrm{CO}_{2}$ in some strains. In most strains, cell-associated radioactivity was located mainly in cold TCA-soluble (pool metabolites) fractions. About $90 \%$ of the cellassociated radioactivity was present in the pool metabolites fraction in the $\mathrm{M}$ strain, and about $10 \%$ in hot TCA-soluble (nucleic acid--teichoic acid-capsular polysaccharide) fraction. Radioactivity in spent medium and the capsular polysaccharide-containing fraction appeared to be present as taurine in this strain. Radioactivity in the pool metabolites fraction of three of the strains examined did not chromatograph as taurine, indicating that taurine was converted into other cell metabolites. One strain incorporated radioactivity from taurine into cellular macromolecules, thus revealing a heterogeneity of staphylococcal taurine metabolism.
\end{abstract}

\section{INTRODUCTION}

Taurine (2-aminoethane sulphonate) is a $\beta$-amino acid that has a wide distribution and abundance in animals (Jacobsen \& Smith, 1968). However, there is limited information on the occurrence, function and metabolism of taurine in bacteria. Taurine catabolism has been studied in a few different bacteria (see Shimamoto \& Berk, 1979, 1980). Taurine has also been identified as a consitituent of bacterial cell surface polymers. Kelly \& Weed (1965) reported that taurine was present in the cell walls of a variant, or possibly symbiont, of Bacillus subtilis. In the most thoroughly documented example, Liau et al. (1974) and Liau \& Hash (1977) reported taurine to be a constituent of the capsular polysaccharide of Staphylococcus aureus strain M (Melly et al., 1974; Scott, 1969). Also, Ohtomo et al. (1980) have reported the presence of taurine in what are referred to as 'cell surface teichoic acid preparations' from encapsulated $S$. aureus strains.

The M strain capsule confers resistance to phagocytosis upon this organism (Melly et al., 1974; Peterson et al., 1978), and the mechanism of this phenomenon has been studied extensively in this strain (Wilkinson et al., 1979a,b). In one of these studies (Wilkinson et al., 1979a) taurine could not be detected upon analysis of cell walls retaining capsular polysaccharide isolated from the $M$ strain grown in medium free from added taurine. A preliminary study of taurine metabolism (Wilkinson et al., 1981) showed that exogenously

† Present address: Department of Biochemistry, Colorado State University, Fort Collins, Colorado 80523, U.S.A.

Abbreviation: BHI, Brain Heart Infusion. 
supplied radiolabelled taurine could be taken up by the $M$ strain, and that most of the radioactivity was extractable by $0.01 \mathrm{M}-\mathrm{HCl}$ at $100^{\circ} \mathrm{C}$ and was dialysable.

We felt it worthwhile to undertake a study of staphylococcal taurine metabolism in view of the potential importance of taurine as a surface component, and lack of knowledge of the metabolism of a molecule to which staphylococci are commonly exposed in their natural environment, the bodies of warm-blooded animals. In this paper we survey aspects of taurine metabolism in various $S$. aureus strains, with more detailed work on the $\mathbf{M}$ strain in particular.

\section{METHODS}

Organisms and cultural conditions. The following $S$. aureus strains were used: encapsulated strain $\mathbf{M}$ and its unencapsulated derivative M variant (Melly et al., 1974; Wilkinson \& Holmes, 1979), encapsulated strain Smith diffuse (Finkelstein \& Sulkin, 1958; Hunt \& Moses, 1958; Wilkinson \& Holmes, 1979), well-known unencapsulated laboratory strain $\mathrm{H}$ and its microencapsulated derivative T (Park et al., 1974; Wu \& Park, 1971), encapsulated strain NS58D (Yoshida, 1971), and encapsulated coagulase-negative strains 11587 and SA222 (King \& Wilkinson, 1981). Although strains 11587 and SA222 are coagulase-negative, DNA-DNA hybridization studies have shown them to be $S$. aureus strains (W. E. Kloos, personal communication). Strain NS58D was kindly provided by Dr C. L. San Clemente, Michigan State University, U.S.A., and strains 11587 and SA222 by Dr W. E. Kloos, North Carolina State University, U.S.A. The strains were grown in either Brain Heart Infusion (BHI) broth (Difco) or PYK medium consisting of $\left(1^{-1}\right): 5 \mathrm{~g}$ Phytone-Peptone (BBL), $5 \mathrm{~g}$ Bacto Yeast Extract (Difco) and $3 \mathrm{~g} \mathrm{~K}_{2} \mathrm{HPO}_{4}$, $\mathrm{pH} 7 \cdot 2$. Inocula were $1 \%$ (v/v) from overnight cultures. Cultures were grown with shaking (200 r.p.m.) at $37^{\circ} \mathrm{C}$ for $18 \mathrm{~h}$.

Uptake and distribution of $[1,2-14 \mathrm{C}]$ taurine. Organisms were grown in $25 \mathrm{ml}$ medium in a $50 \mathrm{ml}$ Erlenmeyer flask supplemented with $1.0 \mu \mathrm{Ci}[1,2-14 \mathrm{C}]$ taurine (specific activity $56.08 \mathrm{mCi} \mathrm{mmol}^{-1} ; 2.08 \mathrm{GBq} \mathrm{mmol}^{-1}$; New England Nuclear). BHI medium contains about $0.3 \mathrm{~mm}$-taurine, whereas taurine was not detected in PYK medium upon amino acid analysis of acid-hydrolysed powders (Wilkinson et al., 1981; R. Greenburg, U.S.D.A. Laboratories, Philadelphia, Pa., U.S.A., personal communication). Unlabelled taurine was added to PYK medium to a concentration of $0.1 \mathrm{~mm}$. Cells were harvested by centrifugation at $13800 \mathrm{~g}$ for $10 \mathrm{~min}$ at $4{ }^{\circ} \mathrm{C}$ and were resuspended in $5 \mathrm{ml}$ distilled water. The cells were fractionated by the procedure of Park \& Hancock (1960) as modified by Wilkinson \& White (1973). The fractions have been designated as follows (Park \& Hancock, 1960): cold TCA-soluble, pool metabolites fraction; ethanol-soluble, lipid fraction; hot TCA-soluble, nucleic acidteichoic acid-capsular polysaccharide fraction; trypsin-soluble, protein fraction; trypsin-insoluble, peptidoglycan fraction. To determine uptake of radioactivity, duplicate $0.1 \mathrm{ml}$ samples of uninoculated growth medium, spent growth medium and resuspended cells were counted (Saleh \& White, 1979). After each extraction in the cell fractionation scheme, the pellets were resuspended in $5 \mathrm{ml} \mathrm{H}_{2} \mathrm{O}$, and duplicate $0.1 \mathrm{ml}$ samples were counted, except for the final peptidoglycan pellet which was resuspended directly in scintillation cocktail. Samples were counted in $10 \mathrm{ml}$ Beckman GP Scintillation Cocktail (Beckman Instruments) in a Beckman LS-8100 scintillation counter.

Identification of radioactivity. Portions (3-5 $\mathrm{ml}$ ) of fractions containing the greatest amounts of radioactivity, i.e., spent growth medium, cold TCA, and hot TCA-soluble fractions, were dialysed against six changes of $500 \mathrm{ml}$ distilled water in $2 \mathrm{~d}$ at $4{ }^{\circ} \mathrm{C}$. The dialysis tubing had a molecular weight cut-off of about 12000 . Samples were taken for determination of radioactivity before and after dialysis, and the volume in the dialysis bag was measured after dialysis to obtain total radioactivity.

Fractions were also subjected to paper chromotography and TLC. Samples were chromatographed on Whatman no. 1 paper or Eastman Chromagram Sheets (13255 cellulose without fluorescent indicator; Eastman Kodak). Before chromatography, TCA was removed from the cold and hot TCA-soluble fractions by five extractions with ether. When necessary, fractions were concentrated by lyophilization. The hot TCA-soluble fraction was hydrolysed in $4 \mathrm{M}-\mathrm{HCl}$ for $4 \mathrm{~h}$ at $105^{\circ} \mathrm{C}$ and $\mathrm{HCl}$ was removed as described by Wilkinson et al. (1978). Unlabelled taurine was used as a standard and was detected by dipping chromatograms in ninhydrin $(0 \cdot 25 \%, \mathrm{w} / \mathrm{v}$ in acetone) followed by heating at $105^{\circ} \mathrm{C}$ for $5 \mathrm{~min}$. In addition, $\left[1,2^{-14} \mathrm{C}\right]$ taurine plus unlabelled carrier taurine was used as a standard and was detected by counting radioactivity. The solvent systems for paper chromatography included: butan-1-ol/acetic acid/water ( $3: 1: 1$, by vol.), propan-2-ol/formic acid/water (40:5:5, by vol.), and propan-2-ol/95\% ethanol/water ( $50: 50: 15$, by vol.). TLC solvent systems included : pyridine/ethyl acetate/acetic acid/water $(5: 5: 1: 3$, by vol.), butan-1-ol/acetic acid/water $(6: 1: 2$, by vol. $)$, and $95 \%$ ethanol/water $(67: 33, \mathrm{v} / \mathrm{v})$.

After chromatography, the dried chromatograms were cut into strips $25 \mathrm{~mm}$ wide surrounding the origin and extending in $12.5 \mathrm{~mm}$ increments. The strips were placed in scintillation vials, moistened with $0.1 \mathrm{ml}$ distilled water and left at room temperature overnight to dissolve material on the chromatogram. Scintillation cocktail was then added and radioactivity determined. 
Table 1. Uptake of $\left[1,2-{ }^{14} \mathrm{C}\right]$ taurine by $S$. aureus strains

The values represent the average of two experiments with strains M, NS58D and SA222 and are the results of one experiment with the other strains.

\begin{tabular}{|c|c|c|c|c|c|}
\hline \multirow[b]{2}{*}{ Strain } & \multicolumn{3}{|c|}{$10^{-6} \times$ Radioactivity (c.p.m.) } & \multicolumn{2}{|c|}{$\begin{array}{l}\text { Percentage } \\
\text { uptake calculated } \\
\text { using values for: }\end{array}$} \\
\hline & $\begin{array}{l}\text { Uninoculated } \\
\text { medium }\end{array}$ & $\begin{array}{c}\text { Spent } \\
\text { medium }\end{array}$ & $\begin{array}{l}\text { Harvested } \\
\text { cells }\end{array}$ & $\begin{array}{c}\text { Spent } \\
\text { medium }\end{array}$ & $\begin{array}{l}\text { Harvested } \\
\text { cells }\end{array}$ \\
\hline M (encapsulated) & $2 \cdot 28$ & $1 \cdot 56$ & $0 \cdot 34$ & 31 & 15 \\
\hline $\begin{array}{l}\text { M variant } \\
\text { (unencapsulated) }\end{array}$ & $2 \cdot 31$ & 1.37 & 0.45 & 41 & 19 \\
\hline $\begin{array}{l}\mathrm{H} \text { (unencapsulated) } \\
\mathrm{T}\end{array}$ & $2 \cdot 31$ & 1.89 & $0 \cdot 19$ & 18 & 8 \\
\hline (microencapsulated) & $2 \cdot 08$ & 1.68 & $0 \cdot 38$ & 19 & 18 \\
\hline $\begin{array}{l}\text { Smith diffuse } \\
\text { (encapsulated) }\end{array}$ & $2 \cdot 18$ & 0.98 & 0.69 & 55 & 32 \\
\hline $\begin{array}{l}\text { NS58D } \\
\text { (encapsulated) }\end{array}$ & $2 \cdot 64$ & 0.47 & 1.00 & 82 & 38 \\
\hline $\begin{array}{l}11587 \\
\text { (encapsulated) }\end{array}$ & $2 \cdot 40$ & 0.41 & $0 \cdot 19$ & 83 & 8 \\
\hline $\begin{array}{l}\text { SA222 } \\
\text { (encapsulated) }\end{array}$ & $2 \cdot 32$ & 1.86 & $0 \cdot 11$ & 20 & 5 \\
\hline
\end{tabular}

\section{RESULTS}

Uptake of $\left[1,2-{ }^{14}\right.$ C $]$ taurine by various $S$. aureus strains

The organisms were grown overnight in PYK medium in the presence of $\left[1,2-{ }^{14} \mathrm{C}\right]$ taurine and radioactivity taken up was determined by (i) the difference between uninoculated and spent medium, and (ii) direct counting of harvested cells resuspended in water (Table 1). Both the encapsulated and unencapsulated strains took up radiolabelled taurine from the growth medium. Uptake varied from $18-83 \%$ of the taurine supplied amongst the different strains, according to method (i). However, the percentage uptake of radiolabelled taurine appeared to be less when the harvested cells were counted [method (ii)]. The difference in uptake measured by the different methods did not appear to be due to different degrees of quenching between the samples since the $\mathrm{H}$-numbers of all samples were similar. [The $\mathrm{H}$-number is a means of quench correction available on Beckman scintillation counters which works by measuring the Compton edge of photoelectrons generated by an external ${ }^{137} \mathrm{Cs}$ source and comparing the position of that edge between quenched and unquenched samples (Anonymous, 1978).] This raises the possibility that some of the taurine had been metabolized to $\mathrm{CO}_{2}$. No experiments were carried out to confirm or deny this possibility.

By method (i) $30 \%$ or more of the radiolabelled taurine supplied was taken up by the $\mathbf{M}$ and $\mathbf{M}$ variant strains. Similar results were obtained when uptake of taurine was studed in these strains grown in BHI broth. In a separate experiment, 19.6 and 23.2 nmol taurine were taken up per mg dry weight of $M$ and $M$ variant strains, respectively, after overnight growth in PYK medium, as determined by counting cell-associated radioactivity.

\section{Distribution of $\left[1,2-{ }^{14} \mathrm{C}\right]$ taurine taken up among cellular fractions}

The distribution of the radioactivity taken up by the strains is shown in Table 2 . In the $M$ strain the radioactivity was confined mainly to two fractions: the cold TCA-soluble fraction $(86 \%)$ and the hot TCA-soluble fraction $(9 \%)$. The distribution of radioactivity was virtually identical whether the M strain had been grown in PYK or BHI media (data not shown). The M variant strain showed a very similar distribution pattern except that a much lower amount of the radioactivity $(3 \%)$ was present in the hot TCA-soluble fraction. Thus, perhaps somewhat surprisingly, most of the radioactivity taken up by the encapsulated $M$ and unencapsulated $M$ variant strains remained in the pool metabolites fraction. 
Table 2. Distribution of $\left[1,2-{ }^{14} C\right]$ taurine taken up by various $S$. aureus strains among cellular fractions

The values represent the average of two experiments with strains M, NS58D and SA222 and are the results of one experiment with the other strains. For starting amounts of radioactivity see Table 1, harvested cells column.

\begin{tabular}{|c|c|c|c|c|c|c|c|c|c|}
\hline \multirow[b]{2}{*}{ Fraction } & & \multicolumn{8}{|c|}{ Distribution of radioactivity ( $\%$ in fraction) } \\
\hline & Strain ... & $\mathbf{M}$ & $\underset{\text { variant }}{\mathbf{M}}$ & $\mathbf{H}$ & $\mathrm{T}$ & $\begin{array}{l}\text { Smith } \\
\text { diffuse }\end{array}$ & NS58D & 11587 & SA222 \\
\hline Harvested cells & & 100 & 100 & 100 & 100 & 100 & 100 & 100 & 100 \\
\hline Pool metabolites & & 86 & 89 & 99 & 97 & 96 & 12 & 94 & 43 \\
\hline Cold TCA pellet & & 14 & 11 & 1 & 3 & 4 & 88 & 6 & 57 \\
\hline Lipid & & 4 & 8 & 1 & 3 & 3 & 1 & 5 & 41 \\
\hline Ethanol pellet & & 10 & 3 & $<1$ & $<1$ & 1 & 87 & 1 & 16 \\
\hline $\begin{array}{l}\text { Nucleic acid-teic } \\
\text { acid-capsular } \\
\text { polysaccharide }\end{array}$ & hoic & 9 & 3 & $<1$ & $<1$ & $<1$ & 62 & 1 & 10 \\
\hline Hot TCA & & & & & & & & & \\
\hline pellet & & 1 & $<1$ & $<1$ & $<1$ & $<1$ & 25 & $<1$ & 6 \\
\hline Protein & & $<1$ & $<1$ & $<1$ & $<1$ & $<1$ & 8 & $<1$ & 6 \\
\hline $\begin{array}{l}\text { Trypsin pellet } \\
\text { (peptidoglycan) }\end{array}$ & & $<1$ & $<1$ & $<1$ & $<1$ & $<1$ & 17 & $<1$ & $<1$ \\
\hline
\end{tabular}

Table 3. Dialysis of spent medium and cold and hot TCA-soluble fractions from $S$. aureus $M$ strain grown in the presence of $\left[1,2-{ }^{14} \mathrm{C}\right]$ taurine

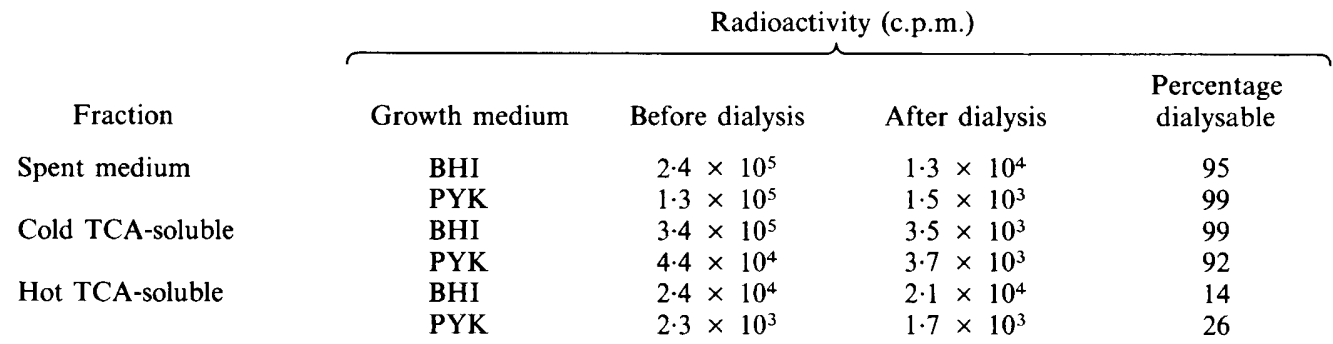

In most of the other strains the majority of the radioactivity was present in the cold TCAsoluble fraction. Radioactivity was not incorporated into the hot TCA-soluble fraction of the $\mathrm{H}$, $\mathrm{T}$ or Smith diffuse strains. The T and Smith diffuse strains have capsular polysaccharides with similar but distinct structures to that of the M strain (Haskell \& Hanessian, 1964; Morse, 1963; Wu \& Park, 1971). Two of the strains had distinctly different distribution patterns. Only a low amount of radioactivity was present in the cold TCA-soluble fraction of strain NS58D $(12 \%)$, and appreciable amounts of label were present in the nucleic acid-teichoic acid-capsular polysaccharide, protein and peptidoglycan fractions. Strain SA222 appeared to incorporate substantial amounts of radioactivity into the lipid and nucleic acid-teichoic acid-capsular polysaccharide fractions. The fact that this organism yielded very 'sloppy' pellets led to some concern about the accuracy of results with this strain.

\section{Identity of cell-associated radioactivity and radioactivity in spent growth medium}

Dialysis of fractions. The spent growth medium and the cold and hot TCA-soluble fractions from the $M$ strain were first dialysed to see whether the radioactivity was present predominantly in high or low molecular weight forms in the fractions (Table 3). Irrespective of whether the $\mathbf{M}$ strain had been grown in BHI or PYK media, more than $90 \%$ of the radioactivity was in a low molecular weight form in both the spent medium and the cold TCA-soluble fraction. This indicates that radioactivity remaining in the spent medium does not represent high molecular 

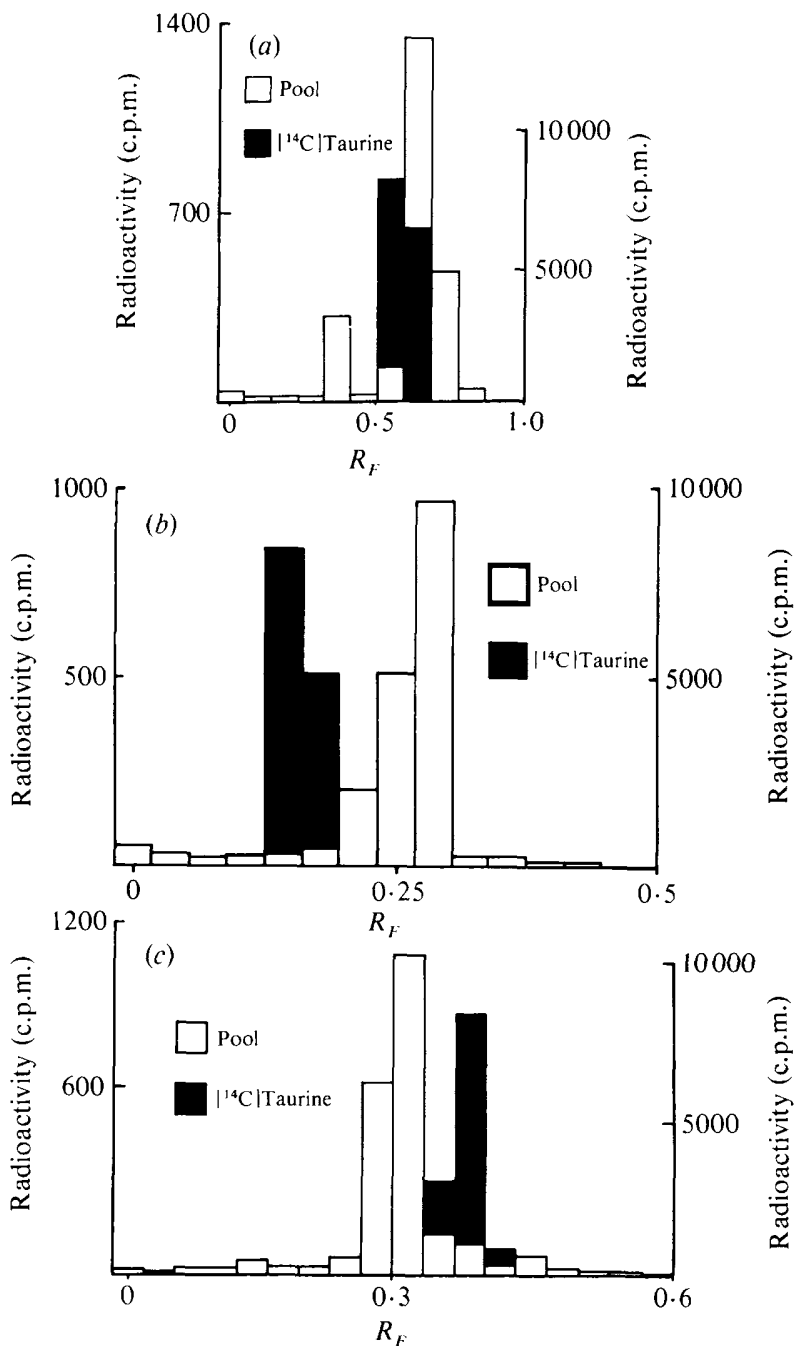

Fig. 1. Chromatography of the cold TCA-soluble fraction from the $\mathbf{M}$ strain. The pool metabolites fraction from $25 \mathrm{ml}$ of culture was obtained as described in Methods. The lyophilized material was dissolved in $0.2 \mathrm{ml} 20 \mathrm{~mm}$ unlabelled taurine and $10 \mu \mathrm{l}$ amounts were chromatographed. A parallel culture was grown without labelled taurine. The unlabelled lyophilized pool metabolites fraction was dissolved in $0.2 \mathrm{ml} 20 \mathrm{~mm}$-taurine containing $0.1 \mu \mathrm{Ci}\left[1,2-{ }^{14} \mathrm{C}\right]$ taurine, and $15 \mu \mathrm{l}$ amounts were chromatographed. In all cases the left-hand axis pertains to chromatography of the pool metabolites fraction of $M$ strain grown in the presence of $\left[1,2-{ }^{14} \mathrm{C}\right]$ taurine and the right-hand axis to $[1,2-$ ${ }^{1+} \mathrm{C}$ ) taurine added to unlabelled pool metabolites fraction. (a) TLC, solvent $95 \%$ ethanol/water $(67: 33$, $\mathrm{v} / \mathrm{v}) ;($ b) paper chromatography, solvent butan-1-ol/acetic acid/water $(3: 1: 1$, by vol.); (c) paper chromatography, solvent propan-2-ol/95\% ethanol/water $(50: 50: 15$, by vol.).

weight capsular polysaccharide released by the organism. Most of the radioactivity in the hot TCA-soluble fraction was non-dialysable, as would be expected if this fraction represents primarily taurine in high molecular weight capsular polysaccharide. The amount of this fraction that was dialysable may represent capsular polysaccharide partially hydrolysed by the hot TCA. Results were similar irrespective of whether the strain had been grown in BHI or PYK media.

Chromatographic analysis. The radioactivity in both the spent medium and acid hydrolysate of the hot TCA-soluble fraction from the M strain chromatographed as one spot with the same $R_{F}$ value as unlabelled or $\left[1,2-{ }^{14} \mathrm{C}\right]$ taurine in four different chromatography systems. Thus it would 
seem that the likely identity of radioactivity in spent medium is unincorporated and/or excreted taurine, while radioactivity in the hot TCA-soluble fraction may represent taurine incorporated into capsular polysaccharide.

Radioactivity in the cold TCA-fraction did not co-chromatograph with authentic taurine. Results with three different chromatography systems are shown in Fig. 1. Similar patterns were seen upon chromatography of the cold TCA-soluble fractions of the $\mathrm{H}$ and NS58D strains which contained greatly differing amounts of label in this fraction $(99 \%$ and $12 \%$ of total cellassociated radioactivity, respectively (see Table 2). Thus it would appear that the majority of the radioactivity in the cold TCA-soluble fraction was not present as taurine but had been converted to another metabolite(s). This result has been confirmed by chromatography of the cold TCAsoluble radioactivity taken up by cell suspensions of the $\mathbf{M}$ strain during short-term incubation with $\left[1,2-{ }^{14} \mathrm{C}\right]$ taurine in buffer (E. Bieber \& B. J. Wilkinson, unpublished observations).

\section{DISCUSSION}

All $S$. aureus strains examined took up significant amounts of radiolabelled taurine from the growth medium (Table 1). In six out of eight of the strains more than $90 \%$ of the cell-associated radioactivity was present in the pool metabolites fraction (Table 2). However, in strain NS58D, radioactivity was present in the nucleic acid-teichoic acid-capsular polysaccharide, protein and peptidoglycan fractions. This suggests that taurine readily enters various metabolic pathways in this strain. In strain SA222 there appeared to be a substantial incorporation of radioactivity into the lipid fraction. Smaller amounts of label were present in this fraction in other strains. A marked discrepancy between taurine uptake measured as depletion from the medium and as radioactivity present in cells (Table 1) also suggested taurine may be degraded to $\mathrm{CO}_{2}$ in some strains.

Radioactivity remaining in the medium in which the $M$ strain had grown appeared to be present as taurine. Most of the radioactivity in the pool metabolites fraction of the $\mathbf{M}$ strain, and other strains examined, did not appear to be present as taurine (Fig. 1). This suggests that staphylococci have a hitherto, we believe, unsuspected capacity to metabolize taurine.

About $10 \%$ of the cell-associated radioactivity in the $M$ strain was located in a cellular fraction soluble in hot TCA, which is believed to represent the capsular polysaccharide (Liau et al., 1974; Wilkinson et al., 1979a).

The radioactivity appeared to be present as taurine in this fraction, suggesting that exogenously supplied taurine can be used in capsular polysaccharide biosynthesis. The capsular polysaccharide of the $S$. aureus $\mathrm{M}$ strain is composed of $N$-acetyl-D-fucosamine, $N$-acetyl-Daminogalacturonic acid and taurine; taurine is believed to be attached to $N$-acetyl-Daminogalacturonic acid via an amide bond (Liau \& Hash, 1977; Liau et al., 1974). No evidence was found for taurine incorporation into the Smith diffuse and T strain capsular polysaccharides (Table 2), which have similar structures to that of the M strain (Haskell \& Hanessian, 1964; Morse, 1963; Wu \& Park, 1971). Taurine has been reported to be present in a "cell surface teichoic acid preparation,' which probably contains capsular polysaccharide, from four serologically distinct encapsulated $S$. aureus strains (Ohtomo et al., 1981). One of these strains, NS58D, incorporated radioactivity from labelled taurine into material soluble in hot TCA, which may represent incorporation into capsular polysaccharide. Similarly, radioactivity from labelled taurine was incorporated into this fraction by encapsulated strain SA222. However, chromatographic analysis of hydrolysed hot TCA-soluble material was not performed. Further precise knowledge about taurine in these capsular polysaccharides is limited since the structures of staphylococcal capsular polysaccharides have been worked out in detail for only a few strains (Wilkinson, 1983).

The observations presented in this paper suggest that both encapsulated and unencapsulated $S$. aureus strains have the capacity to metabolize taurine, and that there is a heterogeneity in that different strains appear to metabolize taurine to varying degrees. Most studies of the bacterial metabolism of taurine have been concerned with taurine degradation in organisms grown on taurine as sole carbon source (Shimamoto \& Berk, 1979, 1980), and even as sole nitrogen and 
sulphur source (Stapley \& Starkey, 1970). To our knowledge, there is currently no information on taurine intermediary metabolism in staphylococci. From studies of taurine degradation in other bacteria we can suggest that taurine may participate in transamination and desulphuration reactions, perhaps leading to sulphite and acetate production in some strains, at which point carbon from taurine may enter a number of metabolic pathways. Sulphite is on the sulphur assimilation pathway in bacteria (Burnell $\&$ Whatley, 1980) and taurine may act as a sulphur source for staphylococci. It is not clear whether staphylococci can biosynthesize taurine, although Ohtomo et al. (1981) have reported an encapsulated $S$. aureus strain with L-cysteic acid decarboxylase activity which is believed to participate in taurine biosynthesis from cysteine in animal tissue (Meister, 1965).

At this time we do not understand the significance of the staphylococcal capacity for taurine metabolism. In animals, the highest concentrations of taurine are found in muscle, heart, brain, retina, blood plasma, breast milk and urine (Meister, 1965). Thus, staphylococci, whose natural environment is the bodies of warm-blooded animals, are continually exposed to this molecule. Recent work in this laboratory has revealed what appears to be a sodium-dependent active transport system for taurine in $S$. aureus (E. Bieber \& B. J. Wilkinson, unpublished observations). In addition to any role taurine might have as a nutrient, it may also be important in the synthesis of highly negatively charged surface molecules which may aid the organisms in resisting host defence systems. The sulphonate group is highly acidic, having a pKa of 1.5 (Jacobsen \& Smith, 1968). Further work will be necessary to appreciate fully the role and significance of taurine metabolism in staphylococci.

We are grateful to David Werkmeister for performing some of the chromotography, and to Jean Nicolaides and Marie Knight for typing the manuscript.

\section{REFERENCES}

ANONYMOUS (1978). Beckman LS 8100 Series Liquid Scintillation Systems. Fullerton, California: Beckman Instruments.

Burnell, J. N. \& Whatley, F. R. (1980). Regulation of sulphur metabolism in Paracoccus denitrificans. Journal of General Microbiology 118, 73-78.

Finkelstein, R. A. \& Sulkin, S. E. (1958). Characteristics of coagulase positive and coagulase negative staphylococci in serum-soft agar. Journal of Bacteriology 75, 339-344.

HaSkell, T. H. Hanessian, S. (1964). The purification and characterization of a new active immunizing polysaccharide prepared from Staphylococcus aureus. Biochimica et biophysica acta 83, 35-41.

Hunt, G. A. \& Moses, A. J. (1958). Acute infection of mice with Smith strain of Straphylococcus aureus. Science 128, 1574-1575.

J ACOBSEN, J. G. \& SMITH, J. L., JR (1968). Biochemistry and physiology of taurine and taurine derivatives. Physiological Reviews 48, 424-509.

Kelly, A. P. \& Weed, L. W. (1965). Taurine as a constituent of a bacterial cell wall. Journal of Biological Chemistry 240, 2519-2523.

King, B. F. \& Wilkinson, B. J. (1981). Binding of human immunoglobulin $\mathrm{G}$ to protein $\mathrm{A}$ in encapsulated Staphylococcus aureus. Infection and Immunity 33, 666-672.

LiAU, D.-F. \& HASH, J. H. (1977). Structural analysis of the surface polysaccharide of Staphylococcus aureus M. Journal of Bacteriology 131, 194-200.

Liau, D.-F., Melly, M. A. \& Hash, J. H. (1974). Surface polysaccharide from Staphylococcus aureus $\mathrm{M}$ that contains taurine, D-aminogalacturonic acid, and D-fucosamine. Journal of Bacteriology 119, 913922.

MeISTER, A. (1965). Intermediary metabolism of the amino acids. In Amino Acid Metabolism, pp. 5931026. New York: Academic Press.

Melly, M .A., Duke, L. J., Liau, D.-F. \& Hash, J. H. (1974). Biological properties of encapsulated Staphylococcus aureus. Infection and Immunity 10, 389-397.

MORSE, S. (1963). Distribution of a surface antigen of Staphylococcus aureus. Nature, London 200, 11261127.

Ohtomo, T., Usui, Y., Yoshida, K., Kawamura, S., Suyama, Y. \& San Clemente, C. L. (1980). Biochemical and immunological properties of cell surface teichoic acid preparations from encapsulated strains of Staphylococcus aureus. Proceedings of the Society for Experimental Biology and Medicine 163, $425-431$.

Ohtomo, T., Yoshida, K. \& San Clemente, C. L. (1981). Effect of bile acid derivatives on taurine biosynthesis and extracellular slime production in encapsulated Staphylococcus aureus S-7. Infection and Immunity 31, 798-807.

PARK, J. T. \& HANCOCK, R. (1960). A fractionation procedure for studies of synthesis of cell wall mucopeptide and of other polymers in cells of Staphylococcus aureus. Journal of General Microbiology 22, 249-258.

Park, J. T., Shaw, D. R. D., Chatterjee, A. N., Mirelman, D. \& Wu, T. (1974). Mutants of staphylococci with altered cell walls. Annals of the New York Academy of Sciences 236, 54-62.

Peterson, P. K., Wilkinson, B. J., Kim, Y., 
Schmeling, D. \& Quie, P. G. (1978). Influence of encapsulation on staphylococcal opsonization and phagocytosis by human polymorphonuclear leukocytes. Infection and Immunity 19, 943-949.

SALEH, F. \& White, P. J. (1979). Metabolism of DD-2,6diaminopimelic acid by a diaminopimelate-requiring mutant of Bacillus megaterium. Journal of General Microbiology 115, 95-100.

Scort, A. C. (1969). A capsulate Staphylococcus aureus. Journal of Medical Microbiology 2, 253-260.

Shimamoto, G. \& Berk, R. S. (1979). Catabolism of taurine in Pseudomonas aeruginosa. Biochimica et biophysica acta 569, 287-292.

Shimamoto, G. \& Berk, R. S. (1980). Taurine catabolism. II. Evidence for the participation of the glyoxylate cycle. Biochimica et biophysica acta 632, 399-407.

Stapley, E. O. \& Starkey, R. L. (1970). Decomposition of cysteic acid and taurine by micro-organisms. Journal of General Microbiology 64, 77-84.

Wilkinson, B. J. (1983). Capsules and slimes. In Staphylococci and Staphylococcal Disease Vol. II. Edited by C. S. F. Easmon \& C. Adlam. London: Academic Press. (in the press).

Wilkinson, B. J. \& Holmes, K. M. (1979). Staphylococcus aureus cell surface: capsule as a barrier to bacteriophage adsorption. Infection and Immunity 23, $549-552$.

Wilkinson, B. J. \& White, P. J. (1973). The effect of antibiotics on synthesis of mucopeptide and teichoic acid by Pediococcus cerevisiae and by a substrain that requires methicillin for growth. Journal of General Microbiology 79, 195-204.
Wilkinson, B. J., Dorian, K. J. \& Sabath, L. D. (1978). Cell wall composition and associated properties of methicillin-resistant Staphylococcus aureus strains. Journal of Bacteriology 136, 976-982.

Wilkinson, B. J., Peterson, P. K. \& Quie, P. G. (1979a). Cryptic peptidoglycan and the antiphagocytic effect of the Staphylococcus aureus capsule: model for the antiphagocytic effect of bacterial cell surface polymers. Infection and Immunity 23, 502508.

Wilkinson, B. J., Sisson, S. P., Kim, Y. \& Peterson, P. K. $(1979 b)$. Localization of the third component of complement on the cell wall of encapsulated Staphylococcus aureus $\mathbf{M}$ : implications for the mechanism of resistance to phagocytosis. Infection and Immunity 26, 1159-1163.

Wilkinson, B. J., Sisson, S. P., Kim, Y., \& Peterson, P. K. (1981). Chemical and biological studies of encapsulated Staphylococcus aureus. In Staphylococci and Staphylococcal Infections, Proceedings of the IV International Symposium on Staphylococci and Staphylococcal Infections, pp. 469-473. Edited by J. Jeljaszewicz. New York: Gustav Fisher Verlag.

Wu, T. C. M. \& PARK, J. T. (1971). Chemical characterization of a new surface antigenic polysaccharide from a mutant of Staphylococcus aureus. Journal of Bacteriology 874-884.

YosHidA, K. (1971). Demonstration of serologically different capsular types among strains of Staphylococcus aureus by the serum-soft agar technique. Infection and Immunity 3, 535-537. 